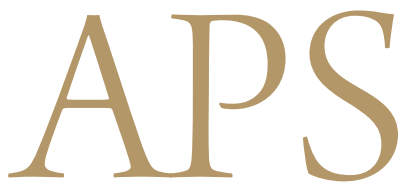

Archives of Plastic Surgery

\title{
The Etiology and Treatment of the Softened Phallus after the Radial Forearm Osteocutaneous Free Flap Phalloplasty
}

\author{
Seok-Kwun Kim, Tae-Heon Kim, Jin-Il Yang, Myung-Hoon Kim, Min-Soo Kim, Keun-Cheol Lee \\ Department of Plastic and Reconstructive Surgery, Dong-A University College of Medicine, Busan, Korea
}

Background The radial forearm osteocutaneous free flap is considered to be the standard technique for penile construction. One year after their operation, most patients experience a softened phallus, so that they suffer from difficulties in sexual intercourse. In this report, we present our experience with phalloplasty by radial forearm osteocutaneous free flap, as well as an evaluation of the etiology and treatment of the softened phallus.

Methods Between March 2005 and February 2010, 58 patients underwent phalloplasty by radial forearm osteocutaneous free flap. Most of their neophallus had been softened subjectively and among them, 12 patients who wanted correction were investigated. We performed repetitive fat injection, artificial dermis grafting, silicone rod insertion, and rib bone with cartilaginous tip graft. Physical examination, plain radiograph, computed tomography, bone scintigraphy, and satisfaction scores were investigated.

Results Most of the participants' penises have been softened after phalloplasty, and the skin elasticity had been also decreased. On plain radiograph, the distal end of the bone was self-rounded; however, the bone shape of the neophallus had no significant interval changes or resorption. Computed tomography showed equivocal density of cortical bone. On bone scintigraphy, the bone metabolism was active at 3 months postoperatively, and remained active 9 years postoperatively.

Conclusions The use of a rib bone with cartilaginous tip graft could be an option for improvement of the softened phallus. Silicon rod insertion is also worth considering for rigidity of the softened phallus. Decreased rigidity due to soft tissue atrophy could be alleviated with repeated fat injection and artificial dermis grafting.

Keywords Free tissue flaps / Penis / Postoperative complications

\author{
Correspondence: Seok-Kwun Kim \\ Department of Plastic and \\ Reconstructive Surgery, Dong-A \\ University College of Medicine, 26 \\ Daesingongwon-ro, Seo-gu, Busan \\ 602-715, Korea \\ Tel: +82-51-240-2807 \\ Fax: +82-51-243-5416 \\ E-mail:sgkim1@dau.ac.kr
}

This article was presented at the 68th Congress of the Korean Society of Plastic and Reconstructive Surgeons on November 4-7, 2010 in Seoul, Korea.

This study was supported by a grant from the Dong-A University research fund.

No potential conflict of interest relevant to this article was reported.

\section{INTRODUCTION}

Ideally, performing a phalloplasty includes a one-stage procedure to construct an aesthetic penis with erogenous and tactile sensation, which enables the patient to void while standing and to have sexual intercourse. In this procedure, the radial forearm osteocu- taneous free flap is one of the most frequently used flaps and considered to be the standard technique in Asia [1]. Unfortunately, over time the neophallus tends to soften. Several techniques have been introduced in an effort to maintain penile stiffness, such as transplants, implants, and external devices. In the radial forearm osteocutaneous free flap, the radius bone is used as a stiffener and

Copyright $\odot 2012$ The Korean Society of Plastic and Reconstructive Surgeons

This is an Open Access article distributed under the terms of the Creative Commons Attribution Non-Commercial License (http://creativecommons.org/

licenses/by-nc/3.0/) which permits unrestricted non-commercial use, distribution, and reproduction in any medium, provided the original work is properly cited.

www.e-aps.org 
to maintain rigidity of the penis. The cause of a softened phallus could be fat tissue resorption or bone resorption. The etiology, in turn, of fat tissue and bone resorption seemed to be decreased metabolism, repetitive friction due to sexual intercourse, and changes in the blood supply. In this context, the authors have attempted to identify the etiology of a softened phallus after the radial forearm osteocutaneous free flap is implemented and to identify a treatment that can restore rigidity.

\section{METHODS}

\section{Patients}

The authors conducted a retrospective study of the transsexuals that were treated at our hospital from March 2005 to February 2010. Fifty-eight patients had a phalloplasty using the radial forearm osteocutaneous free flap according to the authors' technique [2]. The flap size varied according to the condition of donor site-skin, subcutaneous tissue, tattoo, scar, etc.; the radial bone component has a third to a half of the free flap width and 10 to $11 \mathrm{~cm}$ of its length. Among the 58 patients, the 12 who wanted a corrective operation were investigated. The mean age at investigation was 36.2 years old (range, 28 to 45 years old), and the mean postoperative period from the initial operation was 24.8 months (range, 2 to 108 months). The patients' data are presented in Table 1 .

\section{Evaluation}

The circumferences of the glans and penile mid-shaft were measured. All patients underwent a plain radiograph for evaluation of the bony component. Three patients underwent computed tomography to estimate the degree of bone resorption. In two cases, three-phase bone scintigraphy was used to assess metabolic activity. The satisfaction scores after the corrective procedure were also investigated using a questionnaire at postoperative 6 months. The satisfaction score of rigidity and volume had a scale ranging from 1 to 5 , which included 1 ) very poor, 2) poor, 3 ) not good or poor, 4) good, and 5) very good.

\section{Case 1: Silicone rod}

A 42-year-old female-to-male transsexual (FTMTS) patient presented a urethral fistula and softened phallus 9 years postoperatively. The 8-mm-thick subcutaneous tissue had decreased to 5 $\mathrm{mm}$, and the shaft circumference had decreased by $0.8 \mathrm{~cm}$ from $10.8 \mathrm{~cm}$ at its initial construction. A plain radiograph showed no significant resorption of the bone and a computed tomography scan showed normal cortical bone uptake (Fig. 1). Three-phase bone scintigraphy revealed active metabolic activity. The fistula was repaired and a silicone rod was inserted to maintain stiffness of the phallus (Fig. 2).

\section{Case 2: Artificial dermis}

A 44-year-old FTMTS patient who complained of a softened phallus visited our clinic 20 months postoperatively. Physical examination revealed a moderate decrease in skin elasticity and a softened penile shaft, especially of the proximal penis. The shaft circumference had decreased from 11.9 to $11.2 \mathrm{~cm}$. No significant bone resorption was observed on a plain radiograph. Artificial dermis was used to correct the softened phallus.

\section{Case 3: Fat injection}

A 44-year-old FTMTS patient who visited 14 months postoperatively showed decreased soft tissue volume of the neoglans and a palpable bony tip. The shaft circumference had only decreased $0.2 \mathrm{~cm}$. Fat injection was chosen for the corrective procedure (Fig. 3). The fat was cryopreserved and an additional fat injection was performed 3 months after the procedure.

\section{Table 1. Summary of softened phallus patients}

\begin{tabular}{|c|c|c|c|c|c|c|c|c|}
\hline No & Dx & Age (yr) & POD (mo) & Modalities & $\begin{array}{l}\text { Circumference } \\
\text { (cm) }\end{array}$ & Cause & $0 p$ & Satisfaction \\
\hline 1 & GID & 32 & 31 & PEx. X-ray, CT & -1.2 & $\begin{array}{c}\text { Shallow, bony component, } \\
\text { fat absorption }\end{array}$ & Rib bone graft & Very good \\
\hline 2 & GID & 45 & 10 & PEx. X-ray & -0.8 & Fat absorption & Implant & Good \\
\hline 3 & GID & 27 & 13 & PEx. X-ray & -0.9 & Fat absorption & Implant & Very good \\
\hline 4 & GID & 42 & 20 & PEx. X-ray & -0.7 & Fat absorption & Artificial dermis & Good \\
\hline 5 & GID & 40 & $9 \mathrm{yr}$ & PEx. X-ray, 3PBS & -0.8 & Fat absorption & Implant & Very good \\
\hline 6 & GID & 40 & 3 & PEx. X-ray, CT, 3PBS & 0 & Shallow bony component & Rib bone graft & Good \\
\hline 7 & GID & 38 & 9 & PEx. X-ray & -1.2 & Fat absorption & Implant & Good \\
\hline 8 & GID & 28 & 17 & PEx. X-ray & -0.4 & Fat absorption & Fat injection & Dissatisfied \\
\hline 9 & GID & 32 & 2 & PEx. & -0.6 & Fat absorption & Fat injection & Good \\
\hline 10 & GID & 44 & 14 & PEx. & -0.4 & Fat absorption & Fat injection & Very good \\
\hline 11 & GID & 31 & 26 & PEx. X-ray, CT & -1.1 & Fat absorption & Implant & Good \\
\hline 12 & GID & 35 & 44 & PEx. X-ray & -0.8 & Fat absorption & Implant & Good \\
\hline
\end{tabular}


Both immediately postoperative (A) and 1 year postoperative CT scans (B) showed low grade absorption of cortical bone.
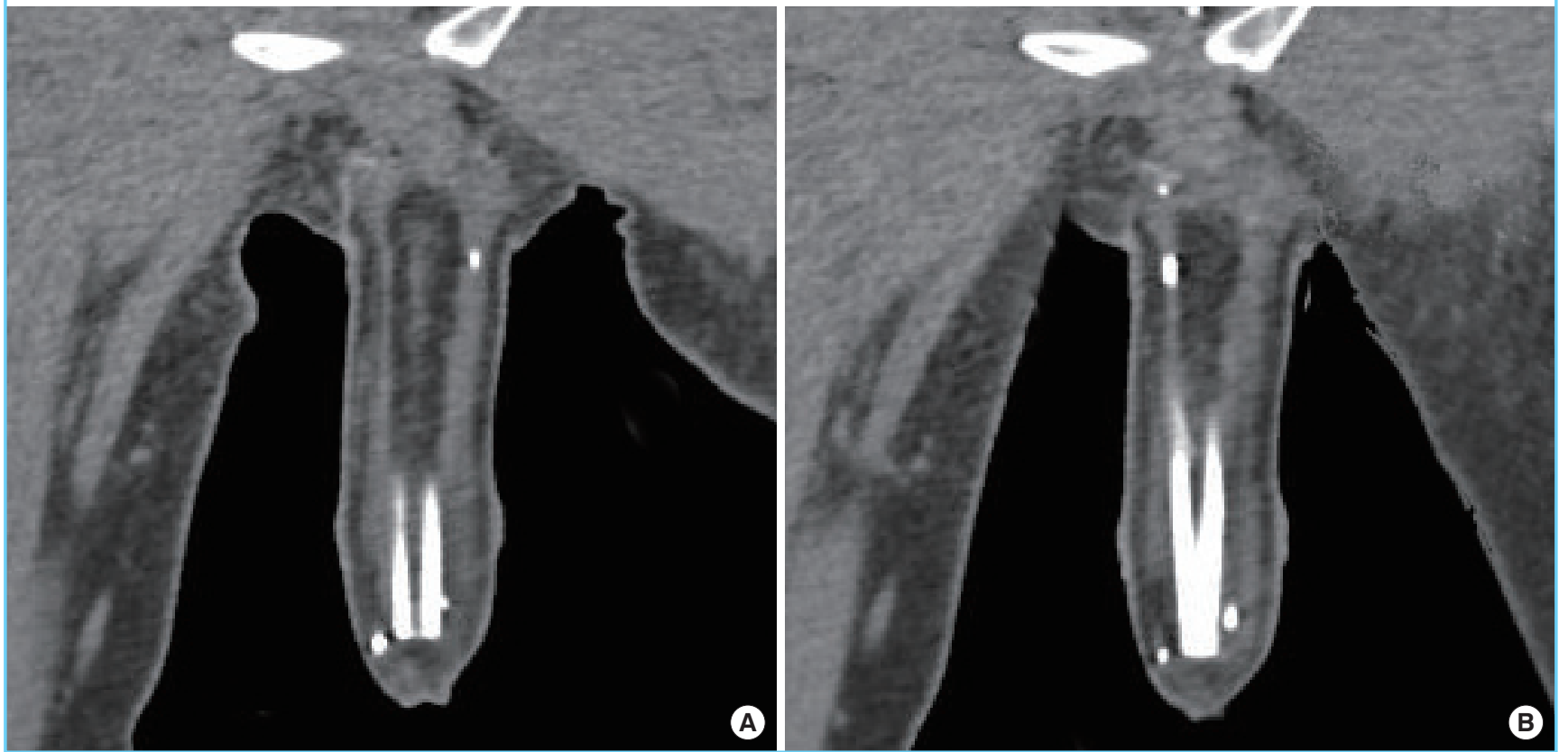

\section{Fig. 2. Insertion of the silicone rod}

A 42-year-old female-to-male transsexual presented with a urethral fistula and a softened phallus. (A) Intraoperative photograph. The silicone rod was inserted into the phallic shaft through a 2 -cm skin incision and the proximal end was fixed to the pubic area. (B) The silicone rod with a soft tip was constructed in compliance with request.

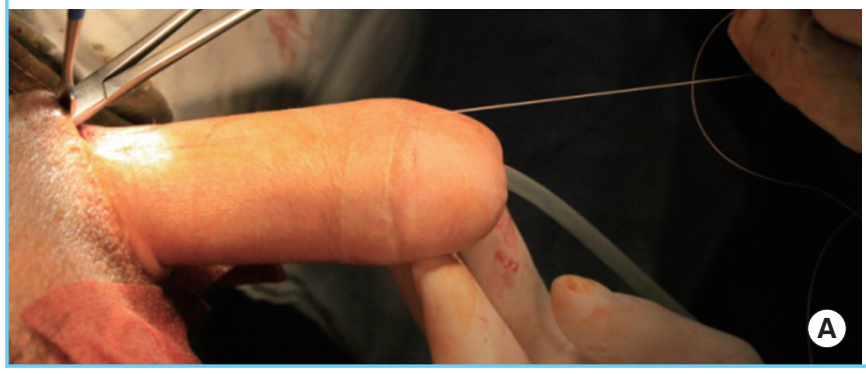

Case 4: Rib bone with cartilaginous tip graft

A 40-year-old FTMTS patient who visited 3 months postoperatively had no changes in skin elasticity or circumference. Plain radiography also showed no significant resorption or fractures. The patient's metabolism was highly active on three-phase bone scintigraphy. However, computed tomography showed some low-grade absorption of the cortical bone, which was thought to be the cause of decreased rigidity originating from the initial operation (Fig. 4). The penile augmentation was performed by a rib bone cartilaginous tip graft (Fig. 5).

\section{RESULTS}

Penile augmentation was performed in 12 patients with softened

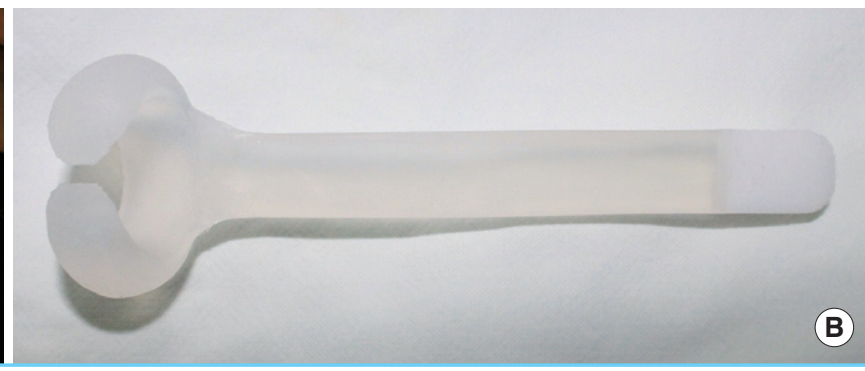

phalli using fat injection, artificial dermis grafts, silicon rod insertion, or rib bone cartilaginous tip grafts. Satisfaction was initially reached in most of the cases. However, in the case of fat injection, most of the injected fat was resorbed and repetitive additional injections were required. In this study, an additional fat injection was performed 1 and 2 months after the initial procedure using the cryopreserved fat. One patient remained dissatisfied despite repetitive fat injections. On physical examination, the thickness of subcutaneous tissue decreased from 8 to $5 \mathrm{~mm}$, and the penile circumference had decreased by $0.74 \mathrm{~cm}$ (range, 0 to 1.2 $\mathrm{cm}$ ) compared to the patient's initial operation. Plain radiograph examination showed a self-rounded bony distal end; however, no significant bone resorption was observed (Fig. 6). There was only one case of bone fracture, for which a rib bone cartilaginous 


\section{Fig. 3. Fat injection}

A 44-year-old female-to-male transsexual presented with palpable bone at the glans tip. (A) Preoperative photograph. A rib bone cartilaginous tip graft had been inserted into phallus previously, but the patient complained of a softened phallus. (B) Three month postoperative photograph after fat injection. The phallus had a little rigidity after a $15 \mathrm{~mL}$ fat injection but the injected fat was mostly resorbed.

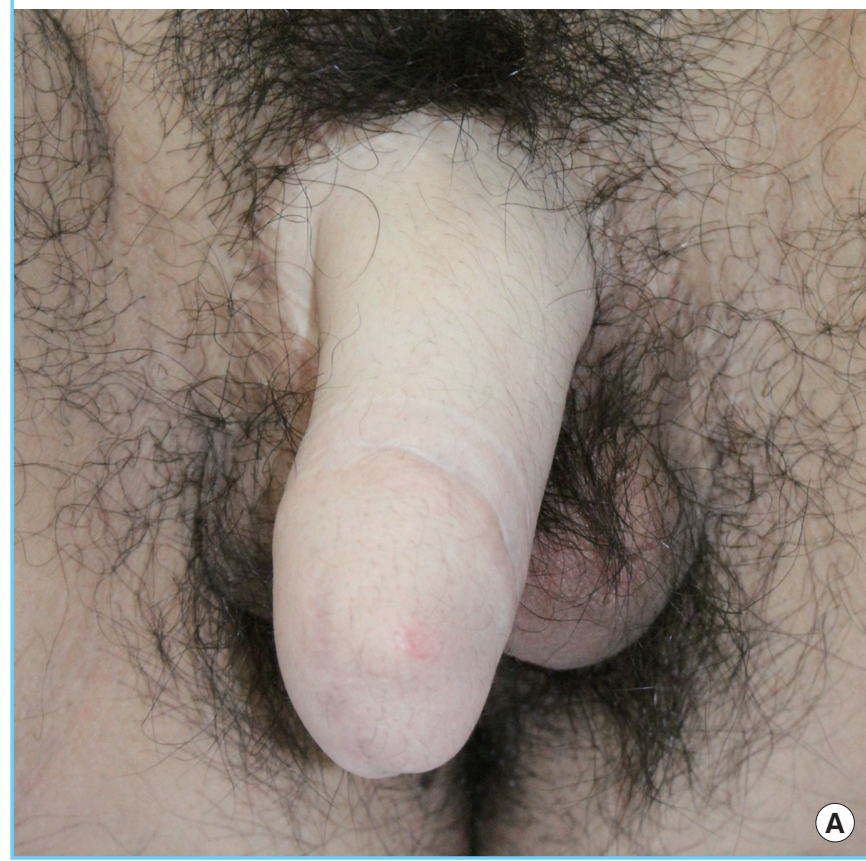

\section{Fig. 4. Three-phase bone scintigraphy}

Three-phase bone scintigraphy of a 42 -year-old female-to-male transsexual showed a urethral fistula and softened phallus. The bone was metabolically active 9 years postoperatively. ANT, anterior; $3 \mathrm{H}$, three hours.

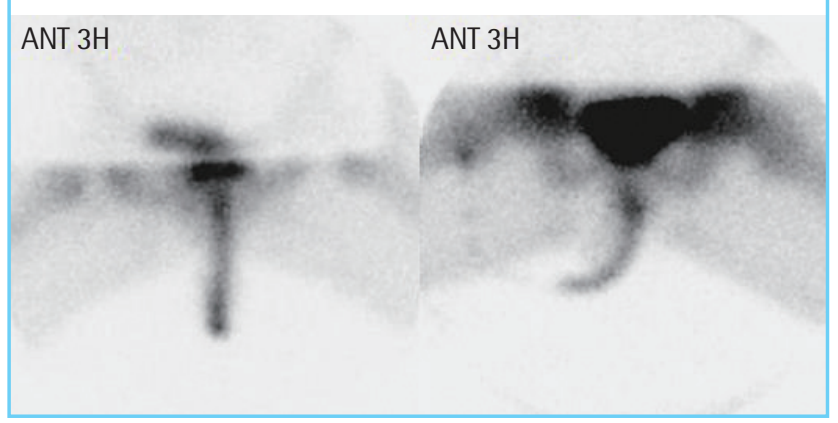

tip graft was performed.

The fat injection group required additional fat injections because the fat was resorbed after 6 months in most of the cases. The artificial dermis group had little resorption compared with the fat injection group; however, the artificial dermis group still had satisfactory results 6 months after the procedure. The rib bone cartilaginous tip graft group had no resorption after 6 months. The follow-up period of these patients was 2 years (range, 1.2 to 3.1 years).

In three cases computed tomography scan showed the Hounsfield unit at 1,387 for the phallic shaft, which was a density

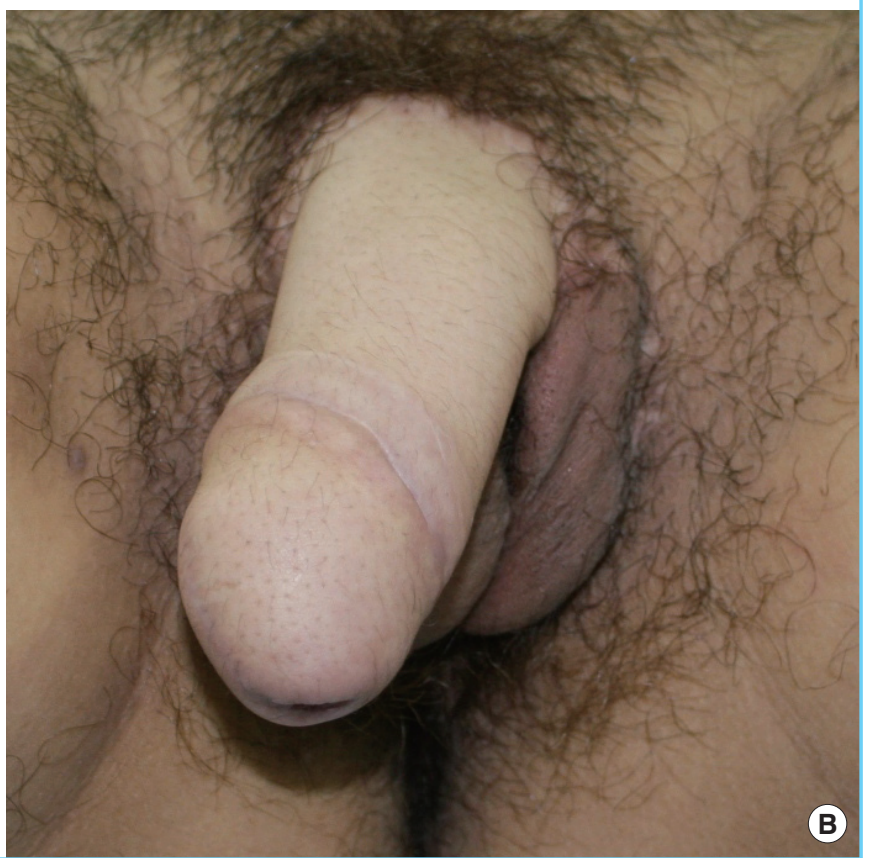

equivalent to normal cortical bone. Three-phase bone scintigraphy was performed on two patients. One patient was 3 months postoperative and revealed active metabolic activity. The other patient was 9 years postoperative and also still had active metabolic activity (Fig. 1). For evaluation of the satisfaction, five questions on satisfaction were asked. The rating consisted of the following: from 1) very dissatisfactory, one point; to 5) very satisfactory correction of shape and rigidity, five points. Four patients scored at 5, 7 patients were at 4, and only one patient who had the secondary corrective procedure scored at 2 (Table 1 ).

\section{DISCUSSION}

Penile construction presents a great challenge for surgeons who perform genital reconstruction. Different techniques have been proposed, including tubed pedicle flaps and pedicled myocutaneous flaps; free tissue transplantation was proposed in the 1980's. Of these flaps, osteocutaneous flaps are known to be feasible for maintaining penile rigidity in a one-stage operation. For phalloplasty specifically, the radial forearm osteocutaneous flap offers several advantages over the other flaps. The radius phallus has good intrinsic rigidity due to its bony component. The flap itself provides superior bone stock due to its volume and strength, and satisfactory penis size can be achieved. Use of the free radial forearm osteocutaneous free flap results in a 


\section{Fig. 5. A 40-year-old female-to-male transsexual patient}

(A) Harvested rib bone, distal end of the rib bone with rib cartilage. Using the bone bender, the shape of the harvested rib bone was made much straighter. (B) Intraoperative view of a rib bone cartilaginous tip graft for augmentation. The rib bone with cartilage was inserted into the phallic shaft. The proximal end of the rib bone was fixed to the periosteum of the pubic bone. (C) Photograph two months postoperatively. Rigidity was obtained by rib bone insertion, and the phallic shaft showed mild curvature.
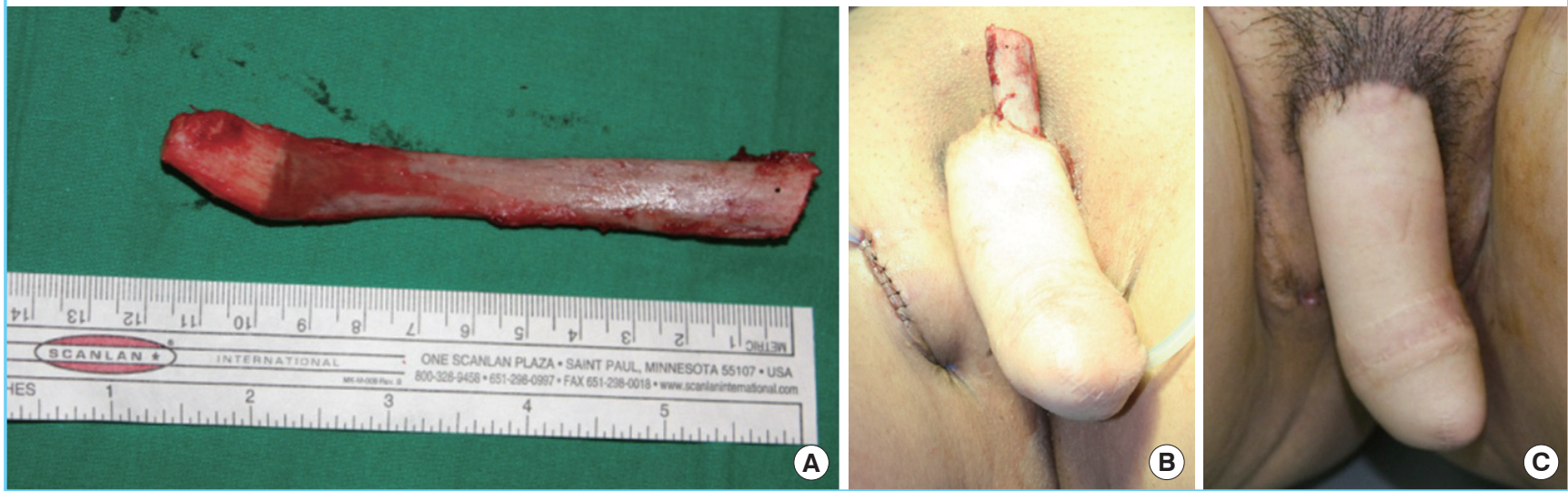

\section{Fig. 6. Plain radiographs of the neophallus}

(A) Immediate postoperative state. Note that the acute angled distal end became self-rounded in shape. (B) Twenty-six months postoperatively. The distal end of the transplanted radial bone rounded itself out.
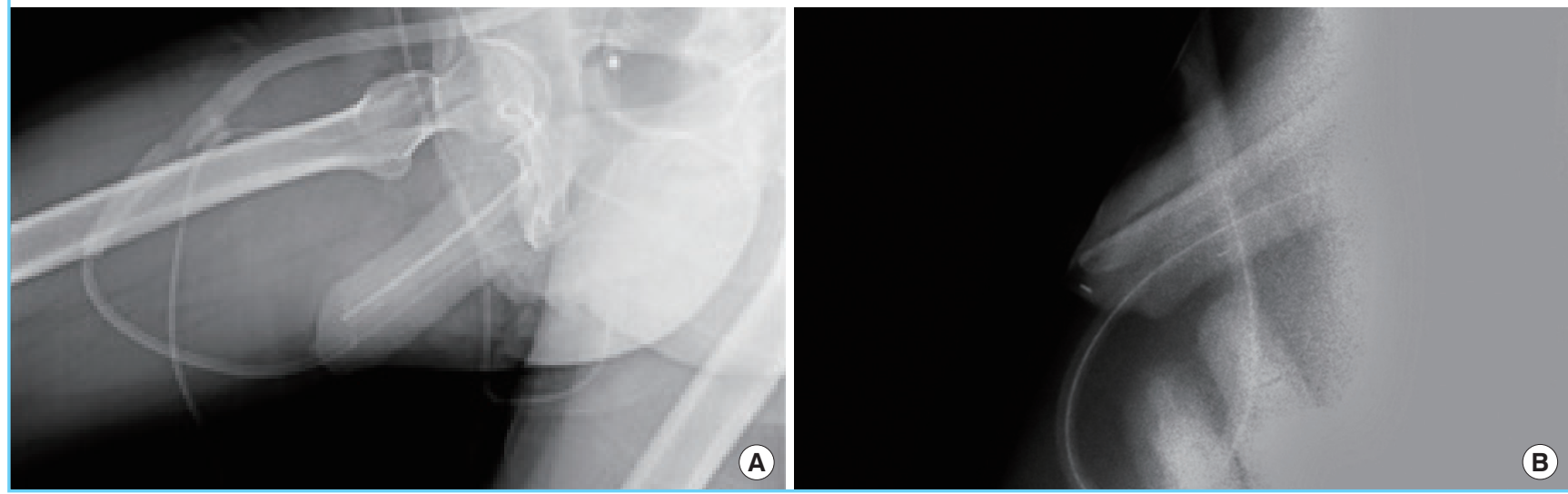

neophallus with a diameter that closely approximates that of an authentic erect penis. The neophallus also has an excellent appearance $[1,3]$.

Various procedures to reconstruct a total phallus have been introduced such as free scapular flap and musculocutaneous latissimus dorsi flap [4-8]. However, these procedures have resulted in a lack of the phallus rigidity; therefore, a prosthesis is needed to obtain the rigidity needed for sexual intercourse.

In our experience, and as previous reports have also affirmed, among the various available techniques, the radial forearm osteocutaneous free flap is the best choice for total phalloplasty in transsexuals. This procedure allows for a vascularized large-sized flap in a one-stage procedure. Furthermore, because urethral fistula can be prevented with a method using a cylindrical siphon technique, which is composed of a labia minoral flap and an anteriorly-based vaginal flap for lengthening the neourethra and enhancing the anastomosis of the urethra to the neourethra, it is considered to be a standard technique for penile construction [9].

However, 1 year postoperatively, there was significant decrease in rigidity of the neophallus, with difficulties in sexual intercourse. There have been some reports on the fate of these flaps or the long-term outcome of penile reconstruction in general. However, there are few reports on the etiology and treatment of a softened phallus.

There are two possible causes of phallus softening. First, soft tissue resorption is the most likely etiology of the softened phallus because of the nature of the osteocutaneous flap, which provides a permanent bony component. Second, resorption of the bony component is another possible cause of a softened phallus. The rate of bone absorption is very low, and a fracture or low- 
grade bone resorption may cause a softened phallus. Although Papadopulos et al. [10] reported decreased metabolic activity at postoperative 2 years as compared with early scintigraphy, in this study, computed tomography and three-phase bone scintigraphy have proven the durability of the bony component of the osteocutaneous free flap. Highly active metabolism means that tissue is well vascularized and vital.

Plain radiographs of the radius bone in the neophalluses showed a robust, calcified bone structure without any evidence of bone resorption or fracture. The computed tomography images showed the cortical substance, which are a characteristic sign of bone viability.

Another cause of softened phallus is soft tissue resorption. None of the procedures related to penile reconstruction are covered by medical insurance in the Republic of Korea. Thus, we have been limited from evaluating the soft tissue configuration using methods such as magnetic resonance imaging because of the payment required. Thus, penile shaft circumferences were chosen as the method for evaluating soft tissue volume. The penile circumference of all patients in this study had decreased significantly at one year postoperatively. This could have been caused by decreased metabolism, repetitive friction due to sexual intercourse, or changes in the blood supply. Most of our patients also had skin redundancy in the neophallus at approximately 12 months postoperatively.

Because patient satisfaction was high in this study, it seems to be important that female-to-male transsexuals have the possibility of sexual activity. Radial forearm osteocutaneous free flap plays a crucial role in phallic reconstruction. Although there was significant volume loss, penile rigidity can be achieved by a penile augmentation procedure after phalloplasty with high patient satisfaction after radial forearm osteocutaneous free flap. Several procedures have been introduced to maintain rigidity [11-13]. Patwardhan said that Bogoraz and Frumken first suggested the use of a stiffener and reported initial satisfaction with costal cartilage and Goodwin and Scott used autogenous costal cartilage rods to substitute for the corpora cavernosa [11]. However, their long-term follow-up showed failure. Chang and Hwang [12] incorporated a cartilage stiffener that could be removed to provide space for future prosthesis placement. Several procedures were attempted in this study to maintain penile rigidity: fat injection, artificial dermis graft, silicone rod insertion, or rib bone graft. Most patients were satisfied initially with their corrective procedure, but in the case of fat injection, most of patients experienced resorption and needed correction by reinjection several times. A somewhat effective and satisfactory result could be achieved for the volume and rigidity of the phallus by repetitive fat injection.
An artificial dermis graft was effective initially, but also showed a small decrease in rigidity due to decreased volume one year after surgery. In our series, the autogenous rib bone cartilaginous tip graft was the best method for rigidity given its definitive increase in rigidity and volume. The curvature of the rib bone cartilaginous tip could be straightened by a bone bender. A silicone rod showed maintenance of rigidity and increase in volume. One patient had an infection after insertion of a silicone rod; thus silicone rod was removed.

Possible causes of the softened phallus included soft tissue resorption and bone resorption. Certainly, soft tissue resorption was the main cause of the decreased rigidity in most of the cases because the rate of bone resorption was extremely low. Although there was some resorption of the bony component, transplanted bone could guarantee good and secure long-term rigidity of the neophallus.

Treatments of the softened phallus that could achieve some effective and satisfactory results include repetitive fat injection and artificial dermis graft in the case of soft tissue atrophy. Rib bone cartilaginous tip graft or silicone rod insertion should be the treatment of choice for both soft tissue atrophy and the bony component problem.

\section{REFERENCES}

1. Monstrey S, Hoebeke P, Selvaggi G, et al. Penile reconstruction: is the radial forearm flap really the standard technique? Plast Reconstr Surg 2009; 124:510-8.

2. Kim SK, Lee KC, Kwon YS, et al. Phalloplasty using radial forearm osteocutaneous free flaps in female-to-male transsexuals. J Plast Reconstr Aesthet Surg 2009;62:309-17.

3. Sadove RC, Sengezer M, McRoberts JW, et al. One-stage total penile reconstruction with a free sensate osteocutaneous fibula flap. Plast Reconstr Surg 1993;92:1314-23.

4. Yang M, Zhao M, Li S, et al. Penile reconstruction by the free scapular flap and malleable penis prosthesis. Ann Plast Surg 2007;59:95-101.

5. Perovic SV, Djinovic R, Bumbasirevic M, et al. Total phalloplasty using a musculocutaneous latissimus dorsi flap. BJU Int 2007;100:899-905.

6. Vesely J, Hyza P, Ranno R, et al. New technique of total phalloplasty with reinnervated latissimus dorsi myocutaneous free flap in female-to-male transsexuals. Ann Plast Surg 2007;58:544-50.

7. Hage JJ, van Turnhout AA. Long-term outcome of metaidoioplasty in 70 female-to-male transsexuals. Ann Plast Surg 2006;57:312-6.

8. Schaff J, Papadopulos NA. A new protocol for complete 
phalloplasty with free sensate and prelaminated osteofasciocutaneous flaps: experience in 37 patients. Microsurgery 2009;29:413-9.

9. Kim SK, Moon JB, Heo J, et al. A new method of urethroplasty for prevention of fistula in female-to-male gender reassignment surgery. Ann Plast Surg 2010;64:759-64.

10. Papadopulos NA, Schaff J, Biemer E. Long-term fate of the bony component in neophallus construction with free osteofasciocutaneous forearm or fibula flap in 18 female-to- male transsexuals. Plast Reconstr Surg 2002;109:1025-30.

11. Patwardhan SK, Shah R, Kulkarni V, et al. Shah's Indian penile prosthesis placement after phallic reconstruction with radial forearm flap. Indian J Urol 2008;24:107-8.

12. Chang TS, Hwang WY. Forearm flap in one-stage reconstruction of the penis. Plast Reconstr Surg 1984;74:251-8.

13. Hoebeke P, de Cuypere G, Ceulemans P, et al. Obtaining rigidity in total phalloplasty: experience with 35 patients. J Urol 2003;169:221-3. 